\title{
FRACTURE MECHANICS SIMPLE CALCULATIONS TO EXPLAIN SMALL REDUCTION OF THE REAL CONTACT AREA UNDER SHEAR
}

\author{
UDC 539.6
}

\begin{abstract}
Michele Ciavarella
Politecnico di Bari, Department of Mechanics, Mathematics and Management, Italy
\end{abstract}

\begin{abstract}
In a very recent paper, Sahli and coauthors [12] (R. Sahli et al., 2018, "Evolution of real contact area under shear", PNAS, 115(3), pp. 471-476) studied the contact area evolution for macroscopic smooth spheres under shear load in presence of adhesion. It was found that contact area $A_{A}$ reduces quadratically with respect to shear load T, i.e. $A=A_{0}-\alpha_{A} T^{2}$, where $A_{0}$ is the contact area with no shearing, and $\alpha_{A}$ is the "area reduction parameter" found to be approximately proportional to $A_{0}^{-3 / 2}$ across 4 orders of magnitude of $A_{0}$. In this note we focus on the smooth sphere/plane contact because we believe that the case of a rough contact requires separate investigations, and we use a known model of fracture mechanics, which contains a fitting parameter $\beta$ which governs the interplay between fractures modes, in order to find very good agreement between the data and the analytical predictions, developing relatively simple equations. The interaction with modes is limited.
\end{abstract}

Key Words: Adhesion, Friction, Fracture Mechanics, Area Reduction, JKR Model

\section{INTRODUCTION}

Adhesion represents a flourishing area of tribology. Several authors are focusing on this topic nowadays for its relevance in different scientific areas, which range from adhesive contact of rough surfaces [1-5] to bioinspired [6-8] and pressure sensitive adhesives [9-11]. A very important topic is the interaction between "adhesion" and "friction". Recently, in a very interesting paper, Sahli et al.[12] make spectacular experiments on the reduction of contact area $A$ upon application of shear force $T$, suggesting it of the form $A=A_{0}-\alpha_{A} T^{2}$, where $A_{0}$ is the force at $T=0$. They suggest scaling laws for coefficient $\alpha_{A}$ of the form $\alpha_{A} \sim$

Received January 08, 2018 / Accepted February 02, 2018

Corresponding author: Michele Ciavarella

Department of Mechanics, Mathematics and Management, Politecnico di Bari, Viale Japigia

E-mail: m.ciava@poliba.it 
$A_{0}{ }^{-3 / 2}$ for individual contacts. Notably, the scaling law is shown to hold over more than 4 orders of magnitude of $A_{0}$, for a plane/plane rough contact as well as for smooth spheres with radii of the order of millimeter, and for a "real" as well as "apparent" contact area. Incidentally, we note that the contact area is still a fugitive concept in contact mechanics [13], due to its fractal evolution, and indeed Sahli et al.[12] do not give any information on the contact radii of asperities, which is a very "resolution-dependent" quantity, as well as the contact area. Therefore, we shall concentrate here on the question of macroscopic contacts, and the corresponding subset of experiments in Sahli et al. [12].

We show here that qualitatively the findings of [12] are predicted by simple Fracture Mechanics arguments for contacts in the presence of adhesion, more in details than what is discussed in [12]. Johnson, Kendall and Roberts (JKR-theory) firstly applied fracture mechanics concepts $[14,15]$ to adhesion between elastic bodies, and this was extended to the presence of tangential force by Savkoor and Briggs [3] who also conducted experiments between glass and rubber similar to [12] but less detailed, and clearly evidenced a reduction of the contact area when tangential load was applied, but the reduction is much less than what is expected by a "brittle model", as indeed is confirmed by [12], which in these respects is therefore not entirely surprising. Johnson [17, 18] and Waters and Guduru [19] have proposed different models to take into account the interplay between two fracture modes, namely I and II (mode III is also present, but marginal) with empirical parameters and phenomenological models to generalize the "brittle" behaviour. We will refer to Johnson [18] in this short communication.

\section{Fracture Mechanics CALCULATIONS}

We will derive here a very simple fracture model in which we retain a circular shape of the contacts, although experiments clearly show an elliptical or even more complex shape, which would make the analysis extremely complex. The energy release sum takes for mixed mode the form (after averaging over the periphery for modes II and III)

$$
G=\frac{1}{2 E^{*}}\left[K_{I}^{2}+\frac{2-v}{2(1-v)} K_{I I}^{2}\right]
$$

where $E^{*}=E /\left(1-v^{2}\right)$ is plane strain elastic modulus, $E$ is Young's modulus and $v$ the Poisson's ratio of the soft material. Here, $K_{I}, K_{I I}$ are Irwin's Stress Intensity Factors. If the surfaces do not slip, $K_{I I}$ is given by [19]

$$
K_{I I}=\frac{T}{2 a \sqrt{\pi a}}
$$

In the absence of tangential force, the equilibrium dictates $G=G_{c}=w$ where $w$ is the surface energy, and the standard JKR Eq. (1) gives contact radius $a_{0}$ for a given normal force $P$

$$
P=\sqrt{8 \pi E^{*} a_{0}^{3} w}-\frac{4 E^{*} a_{0}^{3}}{3 R}
$$


The toughness of the material in mixed-mode conditions is in many phenomenological models a function of ratio $K_{I I} / K_{I}$, i.e. $G_{c}=w f\left(K_{I I} / K_{I}\right)$, where $f\left(K_{I I} / K_{I}\right)=1$ corresponds to the "ideally brittle" fracture, where frictional dissipation is neglected. We follow Johnson ${ }^{1}$ [18] and write $G_{c}$ as

i.e.

$$
\begin{gathered}
G_{c}=w\left[1+\beta Z\left(\frac{K_{I I}^{2}}{K_{I}^{2}}\right)\right] \\
G_{c} \approx w\left[1+\beta Z \frac{T^{2}}{8 \pi E^{*} w a^{3}}\right]
\end{gathered}
$$

where $Z=(2-v) /(2(1-v))$ and we approximate for this step only $K_{I} \approx 2 E^{*} w$.

With applied tangential force $T$, as we have (1), we can restate the JKR mode I problem for a reduced "effective" work of adhesion

i.e.

$$
\begin{gathered}
w_{e f f}=G_{c}-Z \frac{K_{I I}^{2}}{2 E^{*}} \\
w_{e f f}=w[1-(1-\beta) \\
\left.Z \frac{T^{2}}{8 \pi E^{*} w a^{3}}\right]
\end{gathered}
$$

where Eq. (2) has been used. Inserting Eq. (7) into Eq. (3), for the same normal load we require

$$
\sqrt{8 \pi E^{*} a^{3}\left[w-(1-\beta) Z \frac{T^{2}}{8 \pi E^{*} a^{3}}\right]}-\frac{4 E^{*} a^{3}}{3 R}=\sqrt{8 \pi E^{*} a_{0}^{3} w}-\frac{4 E^{*} a_{0}^{3}}{3 R}
$$

Notice that we are conducting our analysis in load control, while displacement control would give different results, as has been shown for work of adhesion independent [20] of the shearing direction. Furthermore, to keep the model simple, we are not considering any dependence of the work of adhesion on the tangential loading, even if such dependence may occur. From Eq. (8), in its general form, it is not evident if this explains the findings in [12]. However, expanding in series for a small change of contact radius $a=a_{0}(1-x)$ leads to (being $x<<1$ )

$$
x=\frac{2 \pi w R\left[(1-\beta) Z \frac{T^{2}}{8 \pi E^{*} a_{0}^{3} w}-1+\sqrt{1-(1-\beta) Z \frac{T^{2}}{8 \pi E^{*} a_{0}^{3} w}}\right]}{\sqrt{8 \pi E^{*} a_{0}^{3} w-(1-\beta) Z T^{2}}-3 \pi w R}
$$

For a sphere, we have $A=\pi a_{0}{ }^{2}(1-x)^{2} \approx A_{0}-2 x A_{0}$, thus $\alpha_{A}=\left(A_{0}-A\right) / T^{2} \approx 2 x A_{0} / T^{2}$. We further expand Eq. (9) for small values of $T$ and obtain

$$
\alpha_{A}=\frac{(1-\beta) Z \pi R}{4\left(\frac{A_{0}}{\pi}\right)^{1 / 2} E^{*}\left[\left(\frac{A_{0}}{\pi}\right)^{3 / 4} \sqrt{8 \pi E^{*} w}-3 \pi R w\right]}
$$

\footnotetext{
${ }^{1}$ Johnson (1996) [18] assumes $v=0$, while we keep the term $Z=(2-v) /(2(1-v))$ in the calculation.
} 
We report in our Fig. 1 the data plotted in Fig. 3 of [12] for smooth contact of spheres with radius $R=[7.06,9.42,24.81] \mathrm{mm}$. Data are reported for $\alpha_{A}$ as a function of $A_{0}$ (circles): they loosely collapse about one line with $\alpha_{A} \sim A_{0}^{-3 / 2}$ which they give as a "guide for eyes" as is here reproduced with the dashed blue line in Fig.1. Using our prediction (10) with material constants $E=1.6 \mathrm{MPa}, w=27 \mathrm{~mJ} / \mathrm{m}^{2}, v=0.5$ and radii $R=[7.06,9.42,24.81] \mathrm{mm}$ (from [12]), we obtain various curves which asymptotically tend to $\alpha_{A} \sim A_{0}{ }^{-5 / 4}$ and with $\beta=0.997$. Unfortunately, Fig. 3 in [12] does not report different symbols for different radii; thus we cannot attempt to compare the dependence on the radius, nevertheless with the same $\beta$ our prediction (10) covers satisfactorily the range of the experimental results.

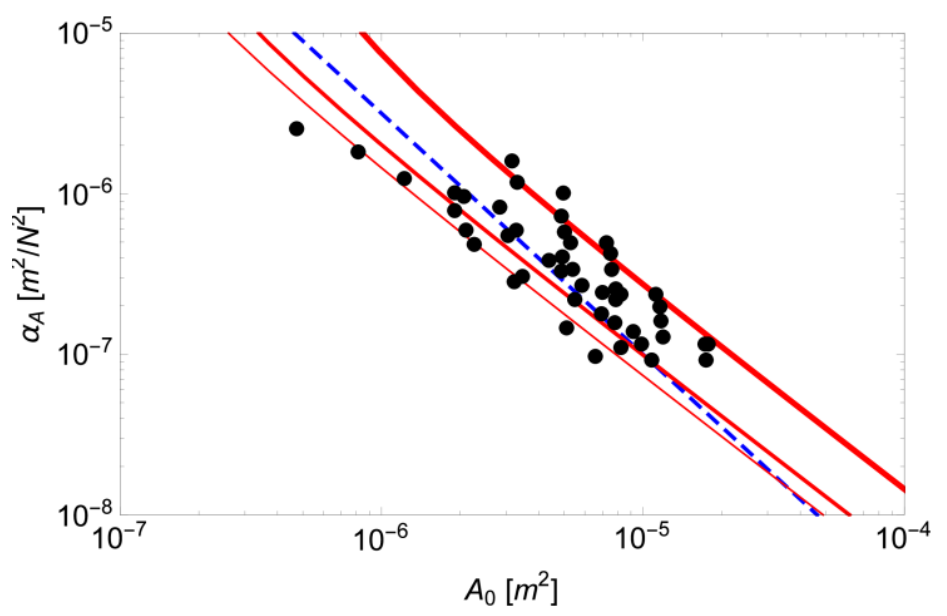

Fig. 1 Comparison of results for area reduction parameter $\alpha_{A}\left[\mathrm{~m}^{2} / \mathrm{N}^{2}\right]$ vs. initial contact area $A_{0}\left[\mathrm{~m}^{2}\right]$ in Fig. 3 of [12] (only for sphere/plane contact) with our prediction (10) for three different radius of the sphere $\mathrm{R}=[7.06,9.42,24.81] \mathrm{mm}$ (solid thick red curves, line thickness increases with radius) and $\beta=0.997$. Dashed line: guide for the eyes with slope $-3 / 2$

\section{CONCLUSIONS}

In this short note, we have used fracture mechanics considerations to estimate the "area reduction parameter" for a smooth macroscopic sphere following the approach of Johnson [16]. We have written the energy release rate in the critical conditions as the sum of the "normal" and "tangential" contribution, where the latter is scaled by a fitting parameter $\beta$. For $\beta=1$ the modes I and II do not interact, and the contact radius is independent of $T$, while for $\beta=0$ the two modes are coupled. The adhesive problem under shear load is seen as an equivalent normal problem but with a reduced work of adhesion. With this model, and expanding for a small variation of the contact area and a small tangential load we have derived analytical predictions for the dependence of the area reduction parameter with respect to $A_{0}$ which asymptotically tend to $\alpha_{A} \sim A_{0}{ }^{-5 / 4}$. The analytical model compared satisfactorily with the experimental data obtained by Sahli and coauthors [12], showing that the interaction with the modes is extremely limited, as the best fit parameter $\beta=0.997$. 


\section{REFERENCES}

1. Fuller, K.N.G., Tabor, D.F.R.S., 1975, The effect of surface roughness on the adhesion of elastic solids, Proceedings of the Royal Society of London A: Mathematical, Physical and Engineering Sciences, 345, 1642.

2. Pastewka, L., Robbins, M.O., 2014, Contact between rough surfaces and a criterion for macroscopic adhesion, Proceedings of the National Academy of Sciences, 111(9), pp. 3298-3303.

3. Ciavarella, M., Papangelo, A., 2018, A generalized Johnson parameter for pull-off decay in the adhesion of rough surfaces, Physical Mesomechanics, 21(1), pp 67-75.

4. Ciavarella, M., Papangelo, A., 2018, A modified form of Pastewka--Robbins criterion for adhesion, The Journal of Adhesion, 94(2), pp. 155-165.

5. Ciavarella, M., Papangelo, A., 2017, A random process asperity model for adhesion between rough surfaces, Journal of Adhesion Science and Technology, 31(22), pp. 2445-2467.

6. Huber, G., Gorb, S., Hosoda, N., Spolenak, R., Arzt, E., 2007, Influence of surface roughness on gecko adhesion, Acta Biomater., 3, pp. 607-610.

7. Pugno, N.M., Lepore. E., 2008, Observation of optimal gecko's adhesion on nanorough surfaces, Biosystems, 94, pp. 218-222.

8. Papangelo, A., Afferrante, L., Ciavarella, M., 2017, A note on the pull-off force for a pattern of contacts distributed over a halfspace, Meccanica, 52(11-12), pp. 2865-2871.

9. Akerboom, S., Appel, J., Labonte, D., Federle, W., Sprakel, J., Kamperman, M.., 2015, Enhanced adhesion of bioinspired nanopatterned elastomers via colloidal surface assembly, Journal of The Royal Society Interface, 12(102), doi:10.1098/rsif.2014.1061.

10. Papangelo, A., Ciavarella, M., 2017, A Maugis-Dugdale cohesive solution for adhesion of a surface with a dimple, Journal of The Royal Society Interface, 14(127), doi: 10.1098/rsif.2016.0996.

11. Papangelo, A., Ciavarella, M., 2018, Adhesion of surfaces with wavy roughness and a shallow depression, Mechanics of Materials, 118, pp. 11-16.

12. Sahli, R., Pallares, G., Ducottet, C., Ben Ali, I.E., Al Akhrass, S., Guibert, M., Scheibert, J., 2018, Evolution of real contact area under shear, Proceedings of the National Academy of Sciences, 115(3), pp. 471-476.

13. Ciavarella, M., Papangelo, A., 2017, Discussion of Measuring and Understanding Contact Area at the Nanoscale: A Review (Jacobs, TDB, and Ashlie Martini, A., 2017, ASME Appl. Mech. Rev., 69 (6), p. 060802), Applied Mechanics Reviews, 69(6), 065502.

14. Johnson, K.L., Kendall, K., Roberts, A.D., 1971, Surface energy and the contact of elastic solids, Proc. R. Soc. Lond. A, 324, pp. 301-313.

15. Popov, V.L., Heß, M., 2015, Method of dimensionality reduction in contact mechanics and friction, Springer, Berlin Heidelberg.

16. Savkoor, A.R., Briggs, G.A.D., 1977, The effect of a tangential force on the contact of elastic solids in adhesion, Proc. R. Soc. Lond. A, 356, pp. 103-114.

17. Johnson, K.L., 1997, Adhesion and friction between a smooth elastic spherical asperity and a plane surface, In Proceedings of the Royal Society of London A453, 1956, pp. 163-179.

18. Johnson, K.L., 1996, Continuum mechanics modeling of adhesion and friction, Langmuir, 12(19), pp. 45104513.

19. Waters, J.F., Guduru, P.R., 2009, Mode-mixity-dependent adhesive contact of a sphere on a plane surface, Proc R Soc A, 466, pp.1303-1325.

20. Popov, V.L., Lyashenko, I.A., Filippov, A.E., 2017, Influence of tangential displacement on the adhesion strength of a contact between a parabolic profile and an elastic half-space, Royal Society Open Science, 4(8), 161010. 